Review

\title{
Perspectives on Inflammatory Breast Cancer (IBC) Research, Clinical Management and Community Engagement from the Duke IBC Consortium
}

Gayathri R. Devi ${ }^{1,2,3 凶}$, Holly Hough ${ }^{4}$, Nadine Barrett ${ }^{1}$, Massimo Cristofanilli ${ }^{5}$, Beth Overmoyer ${ }^{6}$, Neil Spector ${ }^{1,7}$, Naoto T. Ueno ${ }^{8}$, Wendy Woodward ${ }^{9}$, John Kirkpatrick ${ }^{1,10}$, Benjamin Vincent ${ }^{11}$, Kevin P. Williams ${ }^{12}$, Charlotte Finley ${ }^{1}$, Brandi Duff $^{1}$, Valarie Worthy ${ }^{1}$, Shannon McCall ${ }^{1,3}$, Beth A. Hollister ${ }^{2}$, Greg Palmer ${ }^{1,10}$, Jeremy Force ${ }^{1,7}$, Kelly Westbrook ${ }^{1,7}$, Oluwadamilola Fayanju ${ }^{1,2}$, Gita Suneja ${ }^{1,10}$, Susan F. Dent ${ }^{1}$, E. Shelley Hwang ${ }^{1,2}$, Steven R. Patierno ${ }^{1,7}$, P. Kelly Marcom ${ }^{1,7}$

1. Duke Cancer Institute, Duke University;

2. Department of Surgery, Duke University;

3. Department of Pathology, Duke University;

Duke Office of Clinical Research, Duke University;

5. Department of Medicine, Northwestern University;

6. Department of Medical Oncology, Dana-Farber Cancer Institute;

7. Department of Medicine, Duke University;

8. Department of Breast Medical Oncology, University of Texas MD Anderson Cancer Center;

9. Department of Radiation Oncology, University of Texas MD Anderson Cancer Center;

10. Department of Radiation Oncology, Duke University;

11. Division of Hematology/Oncology, University of North Carolina at Chapel Hill;

12. Department of Pharmaceutical Sciences, North Carolina Central University.

$\triangle$ Corresponding author: Gayathri Devi, PhD. Address: 477 MSRB1, 2606 DUMC, Durham, NC 27710. Phone Number and Email: (919) 668-0410, gayathri.devi@duke.edu

(c) Ivyspring International Publisher. This is an open access article distributed under the terms of the Creative Commons Attribution (CC BY-NC) license (https://creativecommons.org/licenses/by-nc/4.0/). See http://ivyspring.com/terms for full terms and conditions.

Received: 2018.11.02; Accepted: 2019.04.12; Published: 2019.06.04

\begin{abstract}
Inflammatory breast cancer (IBC) is an understudied and aggressive form of breast cancer with a poor prognosis, accounting for $2-6 \%$ of new breast cancer diagnoses but $10 \%$ of all breast cancer-related deaths in the United States. Currently there are no therapeutic regimens developed specifically for IBC, and it is critical to recognize that all aspects of treating IBC - including staging, diagnosis, and therapy - are vastly different than other breast cancers. In December 2014, under the umbrella of an interdisciplinary initiative supported by the Duke School of Medicine, researchers, clinicians, research administrators, and patient advocates formed the Duke Consortium for IBC to address the needs of patients in North Carolina (an ethnically and economically diverse state with 100 counties) and across the Southeastern United States. The primary goal of this group is to translate research into action and improve both awareness and patient care through collaborations with local, national and international IBC programs. The consortium held its inaugural meeting on Feb 28, 2018, which also marked Rare Disease Day and convened national research experts, clinicians, patients, advocates, government representatives, foundation leaders, staff, and trainees. The meeting focused on new developments and challenges in the clinical management of IBC, research challenges and opportunities, and an interactive session to garner input from patients, advocates, and community partners that would inform a strategic plan toward continuing improvements in IBC patient care, research, and education.
\end{abstract}

Key words: breast cancer, community engagement, orphan disease, advocacy, patient-centered, inflammatory breast cancer

\section{Introduction}

Substantial progress has been made over the past 10-15 years in understanding inflammatory breast cancer (IBC) tumor biology for improved treatments through commitment from international investigators as part of IBC programs in the United States, the International IBC Consortium (IBC-IC), and partnerships with cancer foundations. However, a major unmet challenge lies in the lack of awareness 
about this breast cancer subtype. IBC presents differently than other types of breast cancer; all tumors are stage III or higher at diagnosis. IBC tumor cells spread rapidly and diffusely within the breast instead of presenting as a clinically apparent mass, which can lead to misdiagnosis and treatment delays. Thus, there is a clear need for further investigations into the mechanisms of aggressive IBC behavior, increased research translation into new treatment regimens for improved patient outcomes, and strengthened community dialogue and engagement to help address the global burden and associated health disparity.

On February 28 ${ }^{\text {th }}$, 2018, which also marked World Rare Disease Day, the Duke Consortium for IBC held its inaugural meeting that comprised attendees $(n=174)$ at the local and national level, including patients, advocates, North Carolina government representatives and stakeholders $(28 \%)$, healthcare providers $(15 \%)$, staff $(15 \%)$, academic research and clinical faculty from local universities and national IBC centers/laboratories (13\%), trainees $(9 \%)$, and individuals who self-identified as 'other' (16\%). The meeting focused on three sessions: a) global partnership in the clinical management of IBC, b) research challenges and opportunities in IBC, and c) engaging advocates and community partners to improve IBC research and education. In keeping with our meeting mission to include patients and their perspectives, several patient stories were interspersed throughout the meeting.

\section{Summary}

Dr. Michal Kastan, Director of the Duke Cancer Institute (DCI), and Dr. Gayathri Devi, Program Director of the Duke Consortium for IBC, welcomed attendees to the campus and noted the importance of collaborative efforts to address this understudied cancer. Dr. Devi provided a brief history of the consortium, which formed in the winter of 2014 when a group of research administrators, patient advocates, and investigators with diverse research and clinical interests from Duke University, the University of North Carolina at Chapel Hill (UNC-CH) and North Carolina Central University (NCCU), came together as part of an interdisciplinary initiative at the Duke University School of Medicine. Based on an analysis of the strengths, weaknesses, challenges and opportunities (SWOT analysis) available at Duke University and the local community to address the unique features and challenges of IBC research and patient care, the group ratified the critical need for a multidisciplinary and cohesive effort in North Carolina, an ethnically and economically diverse state with 100 counties and home to the Research Triangle
Park and multiple institutions of higher education, comprising a major hub for research, medicine, and education. The Duke Consortium for IBC, with over 100 members and an ever-increasing number of partnerships with both local and national patient advocates ranging from grassroots organizations to legislative offices, is committed to the goal of translating research into action. Dr. Kastan reiterated the mission of DCI, which is to transform cancer care through innovative research, integration of academic and clinical missions, and compassionate care, which is in alignment with the goals of the DCI Breast Cancer Program and the IBC consortium efforts. The guest of honor, Dr. Steve Schewel, Mayor of Durham, North Carolina, who is a Duke graduate and Visiting Assistant Professor in the Sanford School of Public Policy, noted that Durham has experienced a high incidence rate of breast cancer in recent years when compared to the rest of North Carolina. He mentioned the widespread health disparities that range from housing, incarceration rates, and employment to nutrition and health, saying "Because of these longstanding disparities in these many areas, it's crucial that we view all of our work here in Durham through a racial equity lens, so I'm especially pleased to be here today to hear you are taking on a disease that disproportionately affects African-American women in Durham." Dr. Schewel also unveiled a map of North Carolina labeled 'Connect NC for IBC' to mark the consortium's initiative to educate and raise awareness about IBC in the local community and across the state.

\section{Global Partnership in Clinical Management of IBC}

The focus of this session was to highlight previous studies using the IBC Consortium datasets to identify the molecular features of IBC [1-4], identify challenges in defining clinical features of IBC, and discuss ongoing clinical trials. Dr. Kelly Marcom, Director of the Breast Cancer Program, opened the clinical session, moderated by Dr. Oluwadamilola "Lola" Fayanju, who is the surgical lead for the IBC clinic, and pathologist Dr. Shannon McCall, with an overview of the Duke Breast Cancer Program, which was launched in 1981 and was the first multidisciplinary breast oncology clinic in the Southeast United States. Over 1,000 new consults are scheduled every year and patients have access to a team of specialists, including genetic counselors and patient navigators. Currently, the Duke Cancer Center sees approximately 15-20 patients diagnosed with IBC per year. Considerably more patients are seen by their community physicians across North Carolina and the southeastern region, including both Virginia and 
South Carolina. The primary goal for the Breast Cancer Program is to establish a flagship clinical service for in-state IBC patients as well as those across the Southeast, currently an unmet need. Dr. Marcom announced the establishment of a new IBC-focused clinic on Tuesdays at the DCI.

Dr. Massimo Cristofanilli, President of the IBC-IC, presented an overview of his program's work, wherein he summarized the clinical features of IBC characterized by rapid onset of aggressive locally advanced disease. As a result of such an aggressive clinical presentation as locally advanced disease, there is no report of a patient with early-stage IBC and 35\% of patients are diagnosed with de novo metastatic disease. From the National Comprehensive Cancer Network Outcomes Database, 673 IBC patients were treated between 1999 and 2009. Of these, 29\% presented with metastatic disease, most were Stage $\mathrm{IIIb}(57 \%)$, and the majority had positive lymph nodes $(81 \%)$. The most frequent site of a first recurrence was bone followed by brain and lung [5]. The data highlight the need for more research in understanding molecular features of this disease and prompted analysis of prospective IBC tissue and blood specimens collected in metastatic settings. Dr. Cristofanilli and his collaborators performed a comprehensive genomic profiling of 53 metastatic IBC tumors, demonstrating a high frequency of genomic alterations in IBC. At least one alteration associated with an FDA-approved therapy or clinical trial was identified in 51/53 tumors, with an average of 2.6 clinically relevant alterations per tumor [6]. An observational analysis of 35 IBC patients ( $80 \%$ had metastatic disease) that failed therapies had plasma analyzed for ctDNA alterations, which were detected in the majority of patients $(94 \%)$ with stage III or IV tumors. The most common mutations were TP53 (49\%), PIK3CA (20\%), ERBB2 (17\%), NOTCH1 (17\%), and ALK (11\%). Genomic information obtained from the analyses was used to select treatments in 11 cases, indicating a potential impact on patient outcomes [7]. Dr. Cristofanilli also mentioned several clinical trials that are currently being conducted, including one at Dana-Farber Cancer Institute where patients with triple-negative IBC receive preoperative treatment with Paclitaxel and Ruxolitinib (+ JAK2 inhibitor). At Northwestern University, patients with HER2-IBC will be recruited for a prospective, single-arm, Phase II study of nab-paclitaxel combined with alpelisib BYL719 following an anthracycline-based treatment regimen. The University of Texas MD Anderson Cancer Center is conducting several clinical trials for both newly diagnosed patients and those with metastatic IBC. Dr. Cristofanilli concluded by indicating that collaboration is critical and, while IBC is not a priority for many, there are organizations dedicated to funding research and improving clinical care of IBC, including the IBC-IC, IBC Research Foundation, Erase IBC, and the IBC Network Foundation.

Dr. Naoto Ueno, Executive Director of the Morgan Welch Inflammatory Breast Cancer Research Program and Clinic at MD Anderson, presented his work targeting epidermal growth factor receptor (EGFR) as a potential therapy for IBC. Sample tissues from 44 IBC patients showed that EGFR was present in $30 \%$ of the cases, which was associated with significantly worse overall survival when compared with EGFR-negative tissues [8]. Additionally, EGFR expression was associated with an increased risk of recurrence. Trastuzumab and cetuximab were developed to target HER2/neu and EGFR, respectively, and have shown efficacy in tumors with these biomarkers [9]. In further study of the EGFR pathway, erlotinib, an EGFR inhibitor, inhibited both proliferation and growth of IBC cells in a xenograft model as well as reversed the mesenchymal phenotype of IBC cells to an epithelial phenotype [10]. Thus, EGFR shows promise because a) hypoactivation of EGFR has been significantly associated with pathological complete response ( $\mathrm{pCR}$ ) in IBC and b) treatment with erlotinib, an EGFR inhibitor, inhibited tumor growth and metastatic progression $[4,10,11]$.

Targeting cancer stem cells may also improve outcomes for IBC patients. Cells that display stem cell properties in normal and malignant breast tissue express the enzyme aldehyde dehydrogenase (ALDH1), which was shown as a predictive factor for the development of systemic metastasis and decreased survival in IBC patients [12]. EGFR regulates IBC cells that express stem cell markers through coclooxygenase-2 (COX-2), whose overexpression is associated with increased cancer cell growth and invasiveness [13]. Nodal, a molecular protein that aids in stem cell maintenance, also mediates EGFR/COX-2-regulated IBC cancer stem cells and, in turn, EGFR regulates Nodal signaling in IBC. Dr. Ueno's lab conducted a Phase II trial with primary HER2-negative IBC patients (19 had triple-negative IBC and 21 had hormone receptor-positive/HER2-negative IBC) who received panitumumab plus neoadjuvant chemotherapy to evaluate $\mathrm{pCR}$ [14]. This treatment combination exhibited the highest $\mathrm{pCR}$ rate ever reported in triplenegative IBC and, thus, a randomized Phase II trial is underway to investigate the role of panitumumab in patients with triple-negative IBC. Future directions for research include enhancing the therapeutic efficacy and further validation of predictive biomarkers of EGFR-targeted therapy in IBC. 
Dr. Beth Overmoyer, Director of the IBC Program at Dana-Farber Cancer Institute, presented the current state of IBC clinical management, highlighting challenges still needing to be addressed. Trimodality therapy is the mainstay of IBC treatment and includes neoadjuvant systemic therapy (NAS) followed by mastectomy and axillary lymph node dissection, radiation to the chest wall and regional lymph nodes, and adjuvant treatment with endocrine therapy (HR+) and/or Trastuzumab or Pertuzumab (HER2+) [15]. The primary challenge is to enable patients to achieve an optimal clinical response to NAS, resulting in maximal reduction in residual cancer within the breast and axillary lymph nodes ( $\mathrm{pCR}$ - negative for tumor presence). This maximizes the ability to complete therapy and is critical for optimal patient outcomes. A study from the National Cancer Database supported this concept and was presented. Patients who had surgical treatment for non-metastatic IBC were identified and after collecting demographic, tumor, and treatment data over time, 5- and 10-year survival rates were highest among patients receiving trimodality treatment. Survival was also significantly higher for patients treated at academic-based programs when compared with those receiving treatment at community programs [15]. The second challenge for physicians is to wait to treat newly diagnosed IBC patients until clinical trials options are discussed. Enrolling IBC patients into clinical trials is challenging, primarily because the clinical signs vary, complicating the diagnosis and often mis-labeling patients as having non-IBC locally advanced breast cancer (LABC). Dr. Overmoyer noted several retrospective studies that used preoperative chemotherapy for the treatment of IBC; however, the patient sample sizes, treatment schemes and median survival varied over time across the studies. When prospective clinical trials were examined, most studies combined IBC with non-IBC patients. An example of the differences in outcomes between the two subtypes of breast cancer was presented. Compared to conventional preoperative chemotherapy, intense dose-dense chemotherapy improved disease-free and overall survival in patients with non-IBC, however, IBC patients experienced a poor prognosis regardless of which chemotherapy scheme they received [16]. From an analysis of SEER data, IBC patients with tumor estrogen receptor and HER2 positivity were associated with better survival than patients with triple-negative IBC [17]. In women with early HER2-positive breast cancer, pertuzumab and trastuzumab, along with standard neoadjuvant chemotherapy, resulted in low rates of symptomatic left ventricular systolic dysfunction in a cardiac safety study [18]. Progression-free survival and disease-free survival at 5-year follow-up show large and overlapping confidence intervals, but supported the primary endpoint $(\mathrm{pCR})$, suggesting that neoadjuvant pertuzumab is beneficial when combined with trastuzumab and docetaxel. Achieving a pCR following NAS could also be an early indicator of long-term clinical outcomes in early-stage HER2-positive breast cancer [19]. Dr. Overmoyer mentioned several potential therapeutic targets for triple-negative IBC, including a) CD44+CD24- cells, which have cancer stem cell-like properties of survival and metastasis [20, 21] and b) the JAK2-STAT3 pathway, a key driver of the CD44+CD24- phenotype and shown to play a role in the pathogenesis of IBC $[22,23]$.

In summary, the common theme identified in this session was the critical need for academic institutions to collaboratively conduct clinical and translational research focused on IBC to help improve treatment for patients.

\section{Research Challenges and Opportunities in IBC}

This session, moderated by medical oncologist Dr. Neil Spector and Dr. Shelley Hwang, Chief of Breast Surgical Oncology and co-lead of the Women's Cancer Program in the DCI, began with Dr. Gayathri Devi discussing the challenge questions she and her team are working to address, such as why do some breast cancer patients with certain subtypes, like IBC, progress more rapidly and become more metastatic? She noted that in aggressive and lesser common subtypes like IBC, there is a paucity of patient biospecimens, patient-derived models, and a lack of incentive for new drug development or clinical trials due to factors related to health disparity, underreporting and misdiagnosis. She then discussed her lab's approach to modeling the unique aspects of IBC progression, focusing on three main points: a) IBC risk factors like race/ethnicity, socioeconomic status and younger age of disease onset have underlying molecular determinants that contribute to health disparity, b) IBC tumor cell clusters/emboli avoid the host immune response and migrate collectively in the lymphatics, and c) IBC tumor cells exhibit a dysregulated adaptive cellular stress response that enhances their survival and leads to drug resistance. She presented data related to the ex vivo tumor emboli simulation model developed in her laboratory that allows for morphometric assessment, along with the ability to image the interior of tumor emboli [24-27]. Using various examples, she presented the advantages of 3D models and patient-derived chronic drug exposure models in IBC research, including high-throughput screening of anti-cancer drugs, 
investigating the role of environmental chemicals in cancer cell proliferation and identifying biomarkers that correlate with resistance to therapy-mediated tumor cell death [11, 28-30]. She also presented recent work conducted in collaboration with Dr. Palmer at the Duke Optical Molecular Imaging and Analysis Core that has led to the novel use of window chambers in the dorsal skin fold of mice to study local migration and invasive characteristics of IBC tumor cells [31]. Dr. Devi then tied in how the preclinical assays are being used in conjunction with spatial distribution models to investigate environmental chemicals that impact IBC incidence and outcomes compared to other types of breast cancer in North Carolina, a collaboration with investigators at the Duke Nicholas School of the Environment [32].

Dr. Wendy Woodward, Chief of the Clinical Breast Radiotherapy Service at MD Anderson, discussed the role the stroma may play in inflammatory changes in IBC. She noted that clinical skin changes are confined to the clinical breast mound in spite of the lack of an anatomic barrier to prevent further spread. This implicates the normal breast in the unique pathogenesis of IBC. The normal breast not involved by tumor in triple-negative breast cancer has a greater number of stem cells, better DNA repair, and is enriched for stem cell gene expression [33]. Normal breast tissue from IBC patients expressed higher numbers of both mammary stem cells and macrophages, and was positively associated with both a tumorigenic stem cell signature and a 79-gene IBC signature [34]. Dr. Woodward's work has included examining the clinical presentation of IBC and how that may be mediated in part by the microenvironment. Her lab has shown that mice who received co-injections of IBC xenografts with mesenchymal stem/stromal cells (MSCs) showed inhibited primary tumor growth, but significantly increased clinical features of skin invasion and development of metastases [35]. Mice injected with MSCs also exhibited increased spontaneous development of metastases following resection of the primary tumor. Thus, there may be crosstalk between macrophages and MSCs, wherein the macrophages educate MSCs to promote an aggressive IBC phenotype. An in vitro culture system has shown that MSCs and macrophages produced higher levels of pro-tumor properties, such as enhanced migration and elevated Il-6 secretion. IBC cells co-cultured with educated MSCs exhibited enhanced invasion that was blocked by anti-IL-6, suggesting that IL-6 is a tumor-promoting mediator and plays a role in migration of MSCs [36]. IBC typically occurs as clusters of cells spread diffusely throughout the breast. Dr. Woodward's lab conducted a brief experiment to determine if normal cells could prime the breast tissue to promote migration. Pro-tumor stroma was used to prime mammary glands and then tumor cells were added, which resulted in scattered emboli that were treatment resistant [37]. Thus, stromal priming appears to promote greater dispersion of tumor cell clusters. Dr. Woodward noted that, from her preliminary work, a) there are significant correlations between the pro-tumor stroma and IBC phenotype, b) infusing normal breast tissue with pro-tumor MSCs and macrophages results in clusters of tumor cells that are IBC-like, and c) pro-tumor MSCs and macrophages promote resistance to radiation therapy.

The meeting also featured several lightning talks. Dr. John Kirkpatrick, Co-Director of the Duke Center for Brain and Spine Metastases, discussed potential collaborative opportunities for research, which include improved chemotherapy delivery, the inflammatory milieu of cell death and improved tumor visibility, and methods for improving the tumor specificity and range of local hyperthermia conferred by laser-induced thermal therapy. Dr. Benjamin Vincent, Assistant Professor in the Division of Hematology/Oncology at UNC-CH, discussed his work surrounding multiple personalized immunotherapeutic strategies and potential collaborative possibilities for IBC. Current research efforts are focused on the development of immunotherapeutic combinations that convert nonresponders to responders and surmount acquired resistance to immunotherapy, thus, identifying biomarkers that predict the patient response to immunotherapy is a high priority. Dr. Kevin Williams, Associate Professor in the Department of Pharmaceutical Sciences at NCCU, discussed existing collaborative research and training partnerships surrounding IBC between Duke University and NCCU. As part of the P20 grant from the National Institutes of Health, pilot projects are being conducted in prostate cancer and IBC, and the Cancer Research Education Program, or C-REP, was created to provide underrepresented minority doctoral students and postdocs with training in clinical research, drug discovery, and biobanking. Drs. Williams and Devi are also collaborating to identify effective therapies for IBC by developing models of IBC for drug screening $[11,30]$.

\section{Connect NC for IBC: Engaging Advocates and Community Partners to Improve IBC Research and Education}

In order to address the need for collaboration among IBC stakeholders across North Carolina and nationally, one of the highlights of this meeting was 
the community engagement session, which brought together basic, translational, and clinical researchers, practicing physicians, patients, advocates, and community stakeholders. In keeping with the meeting mission to engage community members and solicit feedback from patients and advocates, IBC patients shared their stories with attendees.

In particular, Charlotte Finley and Brandi Duff shared moving stories of hope and surviving IBC. Charlotte described waiting for a while when the symptoms appeared before making an appointment with her doctor. By then, her symptoms were worse. When she finally saw a medical oncologist, she asked, "Is there hope?" He smiled and replied, "We've got this." She closed by remembering the happiness she felt post-treatment when seeing that the pathology report indicated no evidence of disease. For Brandi, the anniversary of her IBC diagnosis was precisely one year and a day prior to the meeting date, February $28^{\text {th }}$. Her primary care physician initially prescribed antibiotics for the symptoms, but when things worsened, she underwent numerous tests before being diagnosed by an oncologist. Following chemotherapy, she had a mastectomy and was discharged on her birthday. "So many people commented about how bad it was that I had to be recuperating from surgery on my birthday, but I looked at it as I'm getting to celebrate my $40^{\text {th }}$ birthday cancer free!"

The community engagement session, entitled Conversation Café (facilitated by Dr. Nadine Barrett, Associate Director of Alliances for Community Engagement and Stakeholder Strategy, and Dr. Holly Hough from the Duke Office of Clinical Research) focused on the importance of conversations among all IBC stakeholders to address critical needs in IBC clinical care and outreach. Small groups of diverse stakeholders ranging from 3-8 people engaged in discussion on the following open-ended questions/topics: a) Identify gaps that prevent timely diagnosis and appropriate treatment. b) How can we advocate IBC awareness and work with your local community health providers to become part of your care? c) Identify ongoing grassroots/programmatic efforts in your community. How are you advocating in your community? How are you supporting patients/survivors? and d) How do we partner for philanthropy for research and awareness? Flip chart-size post-it notes were available and group facilitators or someone seated at each table were asked to take notes on the conversation to assist in identifying common themes across the groups. A total of 506 unique responses were recorded and six major themes were identified: barriers to care $(57.7 \%)$, education (16.4\%), outreach/awareness (43.3\%), fundraising (6.3\%), legislative process/priorities $(1.4 \%)$, and 'other' $(3.0 \%)$. This interactive session with the community stakeholders further identified the critical need to address lack of education around IBC at the provider and hospital levels, and the need for better interaction with academic medical centers. There is also a need for observational data on where knowledge gaps exist among providers and how missed diagnoses impact the patient in order to design improved interventions. The IBC community needs more data on where knowledge gaps exist among providers and how missed diagnoses impact patients and their treatment experience in order to design a useful intervention. Social determinants that are known to impact both receipt of care and overall burden of cancer care may play an even larger role in patients with IBC.

\section{Conclusions}

This meeting was a unique opportunity for participants to hear updates from preeminent IBC researchers and clinicians and to network with many different stakeholders in the IBC community [38]. Overall, there is a clear need for collaboration to develop strategic plans and programs for addressing issues surrounding the disease. Measures to improve early detection and diagnosis may have the most benefit when considering prognosis, survival, and treatment costs to patients. Based on strategic planning and findings from our community engagement session, Figure 1 outlines the primary goals for the consortium under each focus area, which include items such as serving more IBC patients via a dedicated clinic within the Duke Breast Oncology Program, further research on immunotherapeutic strategies for IBC, and addressing the need for education among local providers. Clearly, addressing the oncologic and psychosocial needs of IBC patients warrants a multi-faceted approach. This symposium successfully convened stakeholders from across the country who are committed to the holistic treatment of this rare and challenging disease.

\section{Acknowledgments}

The authors wish to thank all patients, advocates, community partners, foundations and trainees who participated in the meeting. We thank the community engagement session facilitators, who included Terry Arnold, Crystal Cates, Jeannine Donahue, Valerie Fraser, Beth Hollister, Phyllis Johnson, Eva May, Amanda McMillan, Ginny Mason, Patricia Moorman, and Patty Spears; Karen Butler, Julie Harbin and Angela Spivey at the Duke Cancer Institute; Scott Behm, Department of Surgery and Laura Hughes at the Duke School of Medicine for 
assistance with graphics and brochure development, administrators from the Department of Surgery and Duke Cancer Institute for volunteering at the meeting; Jennifer McMains, Larisa Gearhart, and Pranalee Patel for editorial assistance during manuscript preparation. We also thank investigators from the International IBC Consortium who sent in their lab overview slides for the monitors. Funding support in part by the Duke University School of Medicine Interdisciplinary Colloquium Grant (GRD), a gift from the IBC Network Foundation, Office of the Chancellor for Health Affairs at Duke University, Duke Departments (Surgery, Medicine, Pathology) and the Duke Cancer Institute.
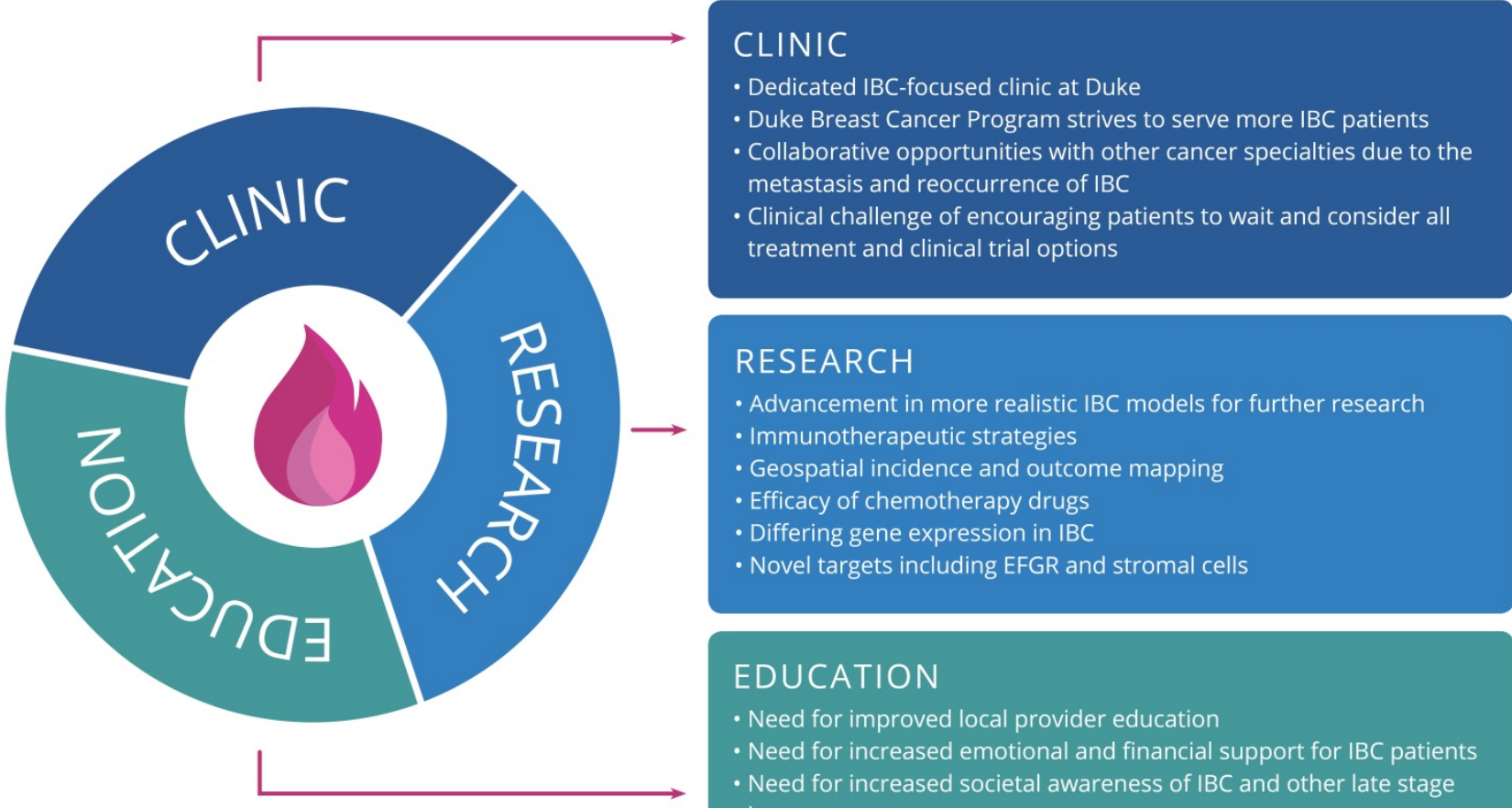

\section{RESEARCH}

- Advancement in more realistic IBC models for further research

- Immunotherapeutic strategies

- Geospatial incidence and outcome mapping

- Efficacy of chemotherapy drugs

- Differing gene expression in IBC

- Novel targets including EFGR and stromal cells

\section{EDUCATION}

- Need for improved local provider education

- Need for increased emotional and financial support for IBC patients

- Need for increased societal awareness of IBC and other late stage

breast cancers

Figure 1. The primary goals for each focus area of the Duke Consortium for IBC.

\section{Competing Interests}

The authors have declared that no competing interest exists.

\section{References}

1. Van Laere SJ, Ueno NT, Finetti P, Vermeulen P, Lucci A, Robertson FM, et al. Uncovering the molecular secrets of inflammatory breast cancer biology: an integrated analysis of three distinct affymetrix gene expression datasets. Clin Cancer Res. 2013; 19: 4685-96.

2. Woodward WA, Cristofanilli M, Merajver SD, Van Laere S, Pusztai L, Bertucci $\mathrm{F}$, et al. Scientific Summary from the Morgan Welch MD Anderson Cancer Center Inflammatory Breast Cancer (IBC) Program 10(th) Anniversary Conference. J Cancer. 2017; 8: 3607-14.

3. Bertucci F, Finetti P, Colpaert C, Mamessier E, Parizel M, Dirix L, et al. PDL1 expression in inflammatory breast cancer is frequent and predicts for the pathological response to chemotherapy. Oncotarget. 2015; 6: 13506-19.

4. Bertucci F, Ueno NT, Finetti P, Vermeulen P, Lucci A, Robertson FM, et al. Gene expression profiles of inflammatory breast cancer: correlation with response to neoadjuvant chemotherapy and metastasis-free survival. Ann Oncol. 2014; 25: 358-65.

5. Matro JM, Li T, Cristofanilli M, Hughes ME, Ottesen RA, Weeks JC, et al. Inflammatory breast cancer management in the national comprehensive cancer network: the disease, recurrence pattern, and outcome. Clin Breast Cancer. 2015; 15: 1-7.

6. Ross JS, Ali SM, Wang $\mathrm{K}$, Khaira D, Palma NA, Chmielecki J, et al Comprehensive genomic profiling of inflammatory breast cancer cases reveals a high frequency of clinically relevant genomic alterations. Breast Cancer Res Treat. 2015; 154: 155-62.

7. Austin L, Paolo F, Sebisanovic D, Siew L, Zapanta A, Schiller BJ, et al. Circulating tumor DND (ctDNA) provides molecular monitoring for inflammatory breast cancer (IBC). San Antonio Breast Cancer Symposium. San Antonio, TX: American Association for Cancer Research; 2014.

8. Cabioglu N, Gong Y, Islam R, Broglio KR, Sneige N, Sahin A, et al. Expression of growth factor and chemokine receptors: new insights in the biology of inflammatory breast cancer. Ann Oncol. 2007; 18: 1021-9.

9. Krejsa C, Rogge M, Sadee W. Protein therapeutics: new applications for pharmacogenetics. Nat Rev Drug Discov. 2006; 5: 507-21.

10. Zhang D, LaFortune TA, Krishnamurthy S, Esteva FJ, Cristofanilli M, Liu P, et al. Epidermal growth factor receptor tyrosine kinase inhibitor reverses mesenchymal to epithelial phenotype and inhibits metastasis in inflammatory breast cancer. Clin Cancer Res. 2009; 15: 6639-48.

11. Sauer SJ, Tarpley M, Shah I, Save AV, Lyerly HK, Patierno SR, et al. Bisphenol A activates EGFR and ERK promoting proliferation, tumor spheroid formation and resistance to EGFR pathway inhibition in estrogen receptor-negative inflammatory breast cancer cells. Carcinogenesis. 2017; 38 : 252-60.

12. Charafe-Jauffret E, Ginestier C, Iovino F, Tarpin C, Diebel M, Esterni B, et al. Aldehyde dehydrogenase 1-positive cancer stem cells mediate metastasis and poor clinical outcome in inflammatory breast cancer. Clin Cancer Res. 2010; 16: 45-55.

13. Wang X, Reyes ME, Zhang D, Funakoshi Y, Trape AP, Gong Y, et al. EGFR signaling promotes inflammation and cancer stem-like activity in inflammatory breast cancer. Oncotarget. 2017; 8: 67904-17.

14. Matsuda N, Wang X, Lim B, Krishnamurthy S, Alvarez RH, Willey JS, et al. Safety and Efficacy of Panitumumab Plus Neoadjuvant Chemotherapy in Patients With Primary HER2-Negative Inflammatory Breast Cancer. JAMA Oncol. 2018: 1207-13

15. Rueth NM, Lin HY, Bedrosian I, Shaitelman SF, Ueno NT, Shen Y, et al. Underuse of trimodality treatment affects survival for patients with inflammatory breast cancer: an analysis of treatment and survival trends from the National Cancer Database. J Clin Oncol. 2014; 32: 2018-24.

16. Untch M, Mobus V, Kuhn W, Muck BR, Thomssen C, Bauerfeind I, et al. Intensive dose-dense compared with conventionally scheduled preoperative chemotherapy for high-risk primary breast cancer. J Clin Oncol. 2009; 27: 2938-45. 
17. Li J, Xia Y, Wu Q, Zhu S, Chen C, Yang W, et al. Outcomes of patients with inflammatory breast cancer by hormone receptor- and HER2-defined molecular subtypes: A population-based study from the SEER program. Oncotarget. 2017; 8: 49370-9.

18. Schneeweiss A, Chia S, Hickish T, Harvey V, Eniu A, Hegg R, et al. Pertuzumab plus trastuzumab in combination with standard neoadjuvant anthracycline-containing and anthracycline-free chemotherapy regimens in patients with HER2-positive early breast cancer: a randomized phase II cardiac safety study (TRYPHAENA). Ann Oncol. 2013; 24: 2278-84.

19. Gianni L, Pienkowski T, Im YH, Tseng LM, Liu MC, Lluch A, et al. 5-year analysis of neoadjuvant pertuzumab and trastuzumab in patients with locally advanced, inflammatory, or early-stage HER2-positive breast cancer (NeoSphere): a multicentre, open-label, phase 2 randomised trial. Lancet Oncol. 2016; 17: 791-800.

20. Van Laere S, Limame R, Van Marck EA, Vermeulen PB, Dirix LY. Is there a role for mammary stem cells in inflammatory breast carcinoma?: a review of evidence from cell line, animal model, and human tissue sample experiments. Cancer. 2010; 116: 2794-805.

21. Dontu G, Al-Hajj M, Abdallah WM, Clarke MF, Wicha MS. Stem cells in normal breast development and breast cancer. Cell Prolif. 2003; 36 Suppl 1: 59-72.

22. Marotta LL, Almendro V, Marusyk A, Shipitsin M, Schemme J, Walker SR, et al. The JAK2/STAT3 signaling pathway is required for growth of CD44(+)CD24(-) stem cell-like breast cancer cells in human tumors. J Clin Invest. 2011; 121: 2723-35.

23. Overmoyer B, Almendro V, Shu S, Peluffo G, Park S, Nakhlis F, et al. Abstract P4-06-01: JAK2/STAT3 activity in inflammatory breast cancer supports the investigation of JAK2 therapeutic targeting. Cancer Res. 2012; 72: P4-06-1-P4--1.

24. Cho EH, Shammas RL, Glener AD, Greenup RA, Hwang ES, Hollenbeck ST. The Impact of Autologous Breast Reconstruction on Body Mass Index Patterns in Breast Cancer Patients: A Propensity-Matched Analysis. Plast Reconstr Surg. 2017; 140: 1121-31.

25. Crawford BM, Shammas RL, Fales AM, Brown DA, Hollenbeck ST, Vo-Dinh T, et al. Photothermal ablation of inflammatory breast cancer tumor emboli using plasmonic gold nanostars. Int J Nanomedicine. 2017; 12: 6259-72.

26. Arora J, Sauer SJ, Tarpley M, Vermeulen P, Rypens C, Van Laere S, et al. Inflammatory breast cancer tumor emboli express high levels of anti-apoptotic proteins: use of a quantitative high content and high-throughput 3D IBC spheroid assay to identify targeting strategies. Oncotarget. 2017; 8: 25848-63.

27. Shammas R, Fales A, Crawford B, Hollenbeck S, Vo-Dinh T, Devi G. Abstract P3-16-02: Nanotheranostics using plasmonic gold nanostars to target inflammatory breast cancer cells and tumor emboli. Cancer Res. 2017; 77: P3-16-02-P3-16-02

28. Williams KP, Allensworth JL, Ingram SM, Smith GR, Aldrich AJ, Sexton JZ, et al. Quantitative high-throughput efficacy profiling of approved oncology drugs in inflammatory breast cancer models of acquired drug resistance and re-sensitization. Cancer Lett. 2013; 337: 77-89.

29. Evans MK, Sauer SJ, Nath S, Robinson TJ, Morse MA, Devi GR. X-linked inhibitor of apoptosis protein mediates tumor cell resistance to antibody-dependent cellular cytotoxicity. Cell Death Dis. 2016; 7: e2073.

30. Oladapo HO, Tarpley M, Sauer SJ, Addo KA, Ingram SM, Strepay D, et al Pharmacological targeting of GLI1 inhibits proliferation, tumor emboli formation and in vivo tumor growth of inflammatory breast cancer cells. Cancer Lett. 2017; 411: 136-49.

31. Evans MK, Brown MC, Geradts J, Bao X, Robinson TJ, Jolly MK, et al. XIAP Regulation by MNK Links MAPK and NFkappaB Signaling to Determine an Aggressive Breast Cancer Phenotype. Cancer Res. 2018; 78: 1726-38.

32. Gearhart-Serna LM, Jayasundara N, Tacam M, Jr., Di Giulio R, Devi GR. Assessing Cancer Risk Associated with Aquatic Polycyclic Aromatic Hydrocarbon Pollution Reveals Dietary Routes of Exposure and Vulnerable Populations. J Environ Public Health. 2018: 5610462.

33. Atkinson RL, Yang WT, Rosen DG, Landis MD, Wong H, Lewis MT, et al. Cancer stem cell markers are enriched in normal tissue adjacent to triple negative breast cancer and inversely correlated with DNA repair deficiency. Breast Cancer Res. 2013; 15: R77.

34. Reddy JP, Atkinson RL, Larson R, Burks JK, Smith D, Debeb BG, et al. Mammary stem cell and macrophage markers are enriched in normal tissue adjacent to inflammatory breast cancer. Breast Cancer Res Treat. 2018; 171: 283-93.

35. Lacerda L, Debeb BG, Smith D, Larson R, Solley T, Xu W, et al. Mesenchymal stem cells mediate the clinical phenotype of inflammatory breast cancer in a preclinical model. Breast Cancer Res. 2015; 17: 42.

36. Wolfe AR, Trenton NJ, Debeb BG, Larson R, Ruffell B, Chu K, et al. Mesenchymal stem cells and macrophages interact through IL-6 to promote inflammatory breast cancer in pre-clinical models. Oncotarget. 2016; 7: 82482-92.

37. Rahal OM, Wolfe AR, Mandal PK, Larson R, Tin S, Jimenez C, et al. Blocking Interleukin (IL)4- and IL13-Mediated Phosphorylation of STAT6 (Tyr641) Decreases M2 Polarization of Macrophages and Protects Against Macrophage-Mediated Radioresistance of Inflammatory Breast Cancer. Int J Radiat Oncol Biol Phys. 2018; 100: 1034-43.

38. Devi GR, Lane W, Hough H, Ingraham K, Arnold T, Barrett N. Current unmet needs in inflammatory breast cancer (IBC) patient care: Analysis of open-ended responses from a community engagement session of clinicians, researchers, advocates, and patients at the Duke Consortium for IBC conference. 11th AACR Conference on The Science of Cancer Health Disparities in Racial/Ethnic Minorities and the Medically Underserved. New Orleans, LA; 2018. 